\title{
A Comparison of CSE and EnergyPlus for Residential Energy Calculations
}

\author{
Neal Kruis ${ }^{1}$, Matthew Larson ${ }^{1}$, Bruce Wilcox ${ }^{2}$, Charles S. Barnaby ${ }^{3}$ \\ ${ }^{1}$ Big Ladder Software, Denver, CO \\ ${ }^{2}$ Independent Consultant, Berkeley, CA \\ ${ }^{3}$ Independent Consultant, Moultonborough, NH
}

\begin{abstract}
CSE (California Simulation Engine) and EnergyPlus are state-of-the-art building energy simulation engines. CSE is used for the underlying calculations for all performance-based energy code compliance analysis for low-rise residential buildings in the U.S. state of California. EnergyPlus is developed through funding by the U.S. Department of Energy, and has historically been used primarily for commercial building applications.

We evaluate these tools with regard to algorithmic methodology, software design, features, accuracy, and runtime in the context of modeling residential building energy use. We compare qualitative differences between the two tools, present quantitative comparisons of accuracy relative to empirical measurements, and show comparative accuracy in the context of prototypical building models.

The tools show comparable accuracy and perform calculations at similar levels of algorithmic fidelity. EnergyPlus has more capabilities for simulation of nontraditional HVAC systems. CSE demonstrates a more careful application of object-oriented software design practices and has significantly better runtime performance (by an order of magnitude).
\end{abstract}

\section{Introduction}

CSE is a relatively unknown yet powerful open-source, whole-building simulation engine. EnergyPlus is a wellknown, powerful, open-source, whole-building simulation engine with a historical focus on commercial building applications. With increasing use cases for building simulation software in the residential sector-including performance-based energy codes, and energy rating systems - increasing attention is placed on the capability of simulation engines to perform quick and accurate simulations of residential buildings. Horowitz et al. (2018) describes many of the use cases for residential building simulations and provides a general overview of the residential simulation landscape. This paper highlights some of the key differences that might inform selection of a whole-building simulation engine for residential applications.

\section{Algorithmic similarities}

It is worth noting some of the similarities of these tools that make them two of the most powerful simulation engines currently available. Both simulation engines utilize rigorous approaches to calculating heat balances within a building, including:

- performing sub-hourly calculations

- using a heat balance model for each zone (with a single, well-stirred thermal zone air node)

- calculating interior and exterior convection and radiation for each surface heat balance on a time-step basis

- passing calculated loads to HVAC systems and correcting the heat balance when the systems are unable to meet the load (resulting in a floating zone temperature)

- calculating inter-zonal and envelope airflow using pressure-based networks

- calculating multi-dimensional heat flow through the building foundations.

Because of these capabilities, both tools are being used in the development of future versions of the "Building Thermal Envelope and Fabric Load Tests" in ASHRAE Standard 140 ("Standard Method of Test for the Evaluation of Building Energy Analysis Computer Programs") (ASHRAE, 2017) with consistent results between the two tools.

\section{Input Similarities}

Both tools operate in batch mode by reading text input files containing a description of the building model and relevant simulation settings, performing relevant calculations, and outputting simulation result files. The individual building blocks of the text input files (e.g., surfaces, internal gains, zones, meters, reports) are referred to as "objects" in both tools - although there are some notable differences between the way objects 
are defined and used. Some of these differences will be highlighted throughout the following sections.

\section{Algorithmic differences}

Although both tools take similarly rigorous approaches to calculating loads, there are some important differences in how the tools approach different aspects of the zonal and surface heat balances. Details of the calculation methods employed by CSE can be found in Barnaby et al. (2013) and in Appendix E of Ferris et al. (2015). The methods used in EnergyPlus are documented in its Engineering Reference (United States Department of Energy (DOE), 2018a).

\section{Long-wave radiant exchange}

Within a zone, the enclosing surfaces exchange longwave radiant heat. The amount of radiation heat transferred among surfaces is based on:

- surface temperatures (raised to the 4th power)

- surface emissivities

- view factors among all enclosing surfaces.

The resulting system of equations required to balance radiant heat exchanges among the enclosing surface creates a computationally intensive, non-linear, densematrix problem.

CSE uses Carroll's method (Carroll, 1980), which employs a simplifying assumption that makes the system of equations much more computationally manageable. Instead of each surface exchanging heat directly with every other surface, they all exchange heat with a central radiant temperature node. This changes the expensive dense matrix calculation into a single simple calculation that requires no specialized linear algebra techniques. It also eliminates the need to calculate view factors.

EnergyPlus uses a method called "scriptF", that calculates the full dense matrix system of equations with view factors. However, unless view factors are preprocessed and input directly by the user, EnergyPlus uses a coarse approximation of view factors based solely on surface area and orientation that is only slightly more rigorous than the Carroll method (Carroll, 1981).

\section{Conduction}

There is some commonality in the way EnergyPlus and CSE calculate conduction through opaque surface:

- Both tools have methods to accommodate steadystate (or "massless") conduction calculations using only overall conductance of the surface.

- Both tools have the ability to use finite difference to calculate one-dimensional conduction through a surface.
CSE has options for either implicit or explicit (the default in CSE) finite difference calculations (where the spatial discretization of the explicit calculations is determined by the software to ensure stability). The use of finite difference allows modeling materials with temperature-dependent conductivity.

EnergyPlus, by default, uses Conduction Transfer Functions (CTFs). These function are fast, but have known limitations for very light and very massive surfaces. Additionally, CTFs cannot handle variable thermal properties (e.g., temperature-dependent conductivity and phase change materials). A finite difference implementation using a Crank-Nicholson scheme with an implicit linear solver is also available in EnergyPlus. EnergyPlus can also model combined heat and moisture transfer into surfaces.

\section{Shading}

The shading of incident solar on building surfaces can be computationally intensive, especially when the calculations are generalized for any structural geometry.

CSE has two shading methods:

1. Analytical algorithms for rectangular fins and overhangs over rectangular windows (that only impact the amount of solar incident on the window-not the parent wall surface).

2. GPU-based pixel counting (Jones and Greenberg, 2011) is supported for calculating the shading of photovoltaic arrays. The same technique will be generalized to all surfaces in an upcoming version of CSE (though it does not currently model shading of opaque building surfaces).

EnergyPlus utilizes computational geometry to calculate the polygonal shadow projections on a surface cast from other nearby surfaces. This method requires algorithms to account for overlapping shadows using boolean operations that only work for the convex subset of polygons.

\section{Software architecture}

Software architecture refers to the underlying design of a project's source code structure. A well designed architecture enhances software usability, flexibility, scalability, and maintainability of the program. While the underlying software architecture is rarely seen by users, it is critical to their experience. As open-source software projects, the source code illustrating the architecture of these programs is freely available online (see The CSE Authors (2018a) and United States Department of Energy (DOE) (2018c)).

EnergyPlus has a relatively loosely defined software architecture. The in-memory representations of simu- 
lation objects each have independently defined structures. Most consistency between object structures is achieved through implicit conventions rather than explicit enforcement by the program architecture. EnergyPlus objects are all stored in simple arrays, again with very little in the form of software architecture to enforce consistency among object arrays.

Furthermore, EnergyPlus source code relies heavily on global state variables (e.g., BeginDayFlag) to operate. This practice has long been viewed as problematic since global variables can be changed anywhere in the code and have unintended consequences elsewhere. To fix problems like this, one must debug the entire program instead of just the isolated components. The use of global state variables also means the EnergyPlus source code is not thread safe (meaning it cannot execute code on multiple processors to take advantage of parallel computing).

Recent efforts including moving EnergyPlus from Fortran to $\mathrm{C}++$ have opened the door to the greater potential of re-architecting the source code (using $\mathrm{C}++$ object-oriented practices). However, the source code is sizable and the overall architecture of the program has experienced little change since the original conversion from Fortran. The reliability and maintainability of the EnergyPlus program remains a high priority for its development and actions are being taken to improve its architecture.

CSE was originally written in $\mathrm{C}$ in a time when memory and performance considerations were much more important. As a result there are some gimmicky (and potentially un-safe) programming techniques employed to minimize memory use. Over time, CSE transitioned to $\mathrm{C}++$ and embraced object-oriented practices.

CSE has a well-defined software architecture: Components of the simulation (e.g. surfaces, internal gains, meters, HVAC systems) are all stored in the program memory as "Records". There is a common "Record" base-class that defines attributes and methods that offer general functionality (e.g., each "Record" has a name and methods for handling object-specific errors). "Records" of a given type are stored in a common container class, called an "Anchor", again providing a set of common methods (e.g. construction/destruction, copying, and iteration). This standard object-oriented approach reduces the development effort for adding new capabilities by minimizing duplication of functionality.

A well designed software architecture emphasizes generalization and minimizes repetitive code, resulting in more efficient source code. As a point of comparison, the compiled executable sizes of CSE and EnergyPlus are $2 \mathrm{MB}$ and $55 \mathrm{MB}$, respectively. Some, but not all, of the the difference in size is related to the additional modeling capabilities in EnergyPlus. While modern computers don't have any trouble storing a $55 \mathrm{MB}$ executable, $55 \mathrm{MB}$ of compiled code can be very difficult to maintain and extend.

\section{Building systems capabilities}

On the whole, EnergyPlus is much more feature-rich than CSE. EnergyPlus has experienced much higher levels of funding to incorporate new features. Many of these features include models for a wide variety of advanced HVAC technologies, implemented primarily for commercial buildings. However, recently there has been an increased focus on making enhancements for residential buildings.

\section{HVAC systems}

Both tools have the capability to represent traditional heating and cooling systems:

- Furnaces (fuel and electric resistance)

- Direct expansion cooling and heating (air conditioners and heat pumps)

There are a number of HVAC systems that EnergyPlus can represent that are not explicitly modeled in CSE, including:

- Variable speed compressor technology for air conditioners and heat pumps

- Evaporative cooling

- Ground source heat pumps

- Hydronic boiler/baseboard systems

- Embedded radiant heating/cooling systems.

EnergyPlus can also model any number of systems serving a single thermal zone.

\section{Air distribution}

In residential buildings the design and location of ducting for air distribution is critical to performance. To capture this performance the simulation engines must be capable of accounting for air leakage (and the corresponding interaction with other mechanical and natural airflows within the building) as well as conduction, convection, and radiant exchange with the surrounding environment.

Modeling the effects of ducts in EnergyPlus requires use of its AirflowNetwork capability. EnergyPlus models the pressure throughout the distribution system. This is a more physical representation, however it requires greater input detail, including definitions of all pressure drops within the distributions system characterized by its dimensions (e.g., length and hydraulic diameter of each duct segment) - that are not always available depending on the level of analysis - as well as flow coefficients and exponents for every leakage. This more de- 
tailed characterization of distribution systems is likely more accurate, however it also contributes to longer simulation times.

CSE takes a simplistic approach to modeling leakage in that the duct leakage is set as a constant fraction of the system airflow. Pressure within the ducts is not calculated to determine leakage.

\section{Hot water events}

Many occupant driven events within a housing unit are discrete, on/off, events (e.g., switching lights, running a shower, turning on an oven). These can all be described using a start time, a duration, and a magnitude (e.g., power level or flow rate during the event). For the sake of calculating energy loads, the magnitude of many of these events can be estimated using a time-averaged value (e.g., it is not uncommon to see an occupant schedule with non-integer number of people in a zone during a given hour). Hot water systems, however, are one case where the energy impact is highly non-linear. A high-flow-rate, short-duration event does not have the same impact on energy as a low-flow-rate, long-duration event of the same total draw volume. This is especially true for heat pump water heaters that typically use backup resistance heat for short-duration, high-flow-rate draws.

CSE is able to characterize hot water draws as discrete events using localized 1 minute "sub-sub-hour" timesteps, whereas EnergyPlus requires shorter overall timesteps to represent short duration events.

\section{User Features}

There are some notable differences in how the programs are used that do not necessarily relate to the actual building systems represented in a model. One important, but fairly straightforward difference is the use of unit system: EnergyPlus uses SI units while CSE uses IP units (due to its original, limited application within the United States). The features of both programs are described in their respective user manuals (The CSE Authors (2018b) and United States Department of Energy (DOE) (2018b)).

\section{Input syntax}

The only direct interface to both simulation engines (whether within a user-interface or directly by the user) is through text input files. The design of the input syntax impacts the usability, flexibility, scalability, and maintainability of any software interfacing to the engine at a higher level, including graphical user interfaces.

EnergyPlus's traditional input format is IDF (input data format). IDF describes input objects using a comma separated list of values terminating in a semicolon. Each position in this list correlates to a differ- ent input field for the object (e.g., the sixth value for the ElectricEquipment object represents the design wattage of the equipment). Non-required values left blank or not entered are allowed to default. While this input structure allows for extensible value fields (e.g., repeat the last four fields of the coil object to define performance at different stages), it does not accommodate more complex data structures (e.g., multiple lists of different lengths or nested hierarchical components).

As of its version 8.9.0 release (in March, 2018), EnergyPlus now offers a JSON-based input format called epJSON. This allows for objects to be defined using key/value pairs (rather than the IDF's positional list structure). In this format, any non-required keys that are not defined are allowed to default. JSON itself allows for many of the complex data structures that were not possible in IDF, however, the current implementation of epJSON does not utilize this capability as its data structure is defined by (and generated from) EnergyPlus's input data dictionary (IDD), the same document that defines the structure of IDF input.

When reading IDF or epJSON, EnergyPlus converts everything to in-memory JSON objects, then maps the JSON objects to each simulation object structures using customized source code for each object. This is necessary since there is currently no base-class to map inputs to source code objects in a consistent fashion.

CSE's text file format is unique to CSE and by convention uses the *. cse extension. It uses both key/value pairing and an extensible hierarchical structure. The hierarchical relationship of objects can be implicit (e.g., any GAIN objects defined after a ZONE object will automatically be associated with that zone.), or explicitly linking key values to reference a parent object (e.g., gnZone = "Dwelling").

When reading an input text file, CSE maps the object keys directly to the data members of the corresponding "Record" class in the source code using a consistent mapping structure across all "Record" types.

\section{Automated input manipulation}

CSE's input format also accommodates a built-in parametric preprocessor (used for basic parameter substitution and conditional logic) and contains a set of instruction commands to modify the input between multiple runs without needing to produce an entirely separate input file. While this kind of functionality is not available natively within EnergyPlus similar capabilities are possible through the application of various scripting and middleware frameworks (e.g., EPMacro, eppy, OpenStudio SDK, and Modelkit-see Roth et al. (2018)). 


\section{User-defined behavior}

Some advanced applications of building energy modeling require users to adapt the default runtime behavior of the simulation. Both simulation engines have some capability to override or manipulate aspects of objects throughout the simulation.

EnergyPlus has the Energy Management System (EMS), which allows the user to create "Programs" to override object "Actuators" based on "Sensor" outputs elsewhere in the model ${ }^{1}$. Only a subset of EnergyPlus object inputs can be "actuated" with an EMS program, however any time series report variable can act as a "Sensor". As an example, schedules in EnergyPlus are defined for many inputs that can vary over time (e.g., setpoints, ventilation, internal gains). While schedule objects can only represent variation with time of year, an EMS program can override schedule values with results calculated based on sensor values. Because it is not always clear when a "sensor" value is updated, EMS "Programs" can only be called at specific points throughout the simulation (e.g., beginning of the zone timestep or within the HVAC iteration loop). The following IDF input below shows how one would vary the power of a refrigerator based on zone temperature using EMS.

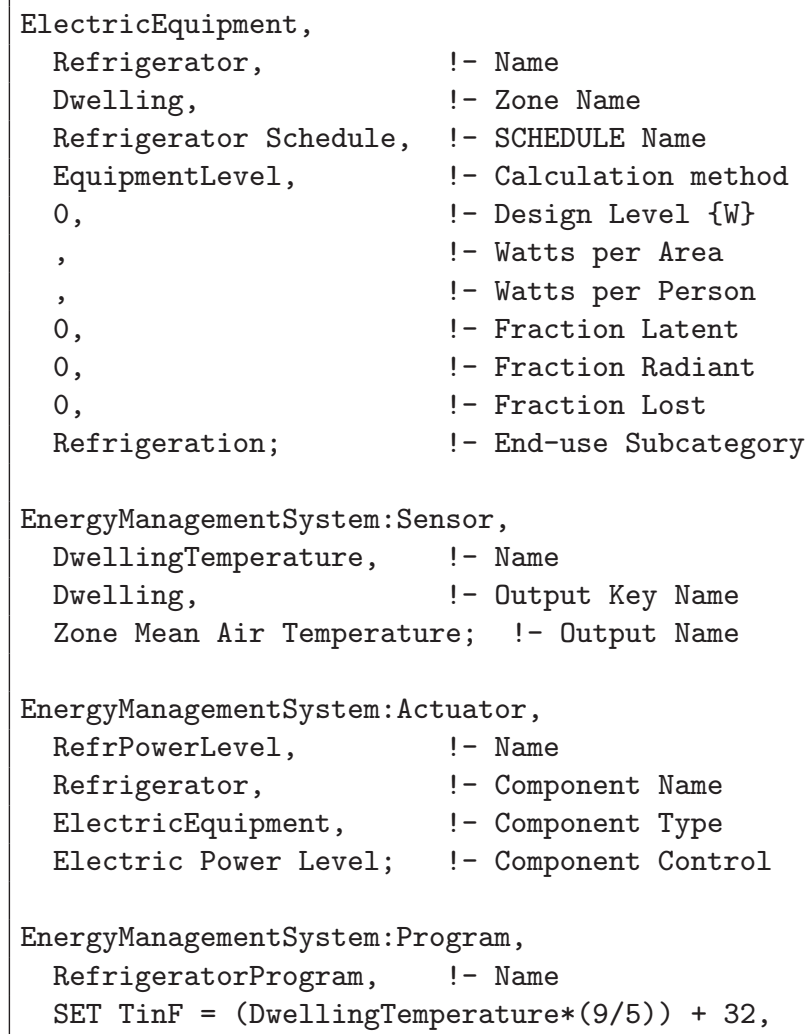

${ }^{1}$ EnergyPlus also provides the External Interface that allows external programs to read EnergyPlus "Sensors" and override object "Actuators".

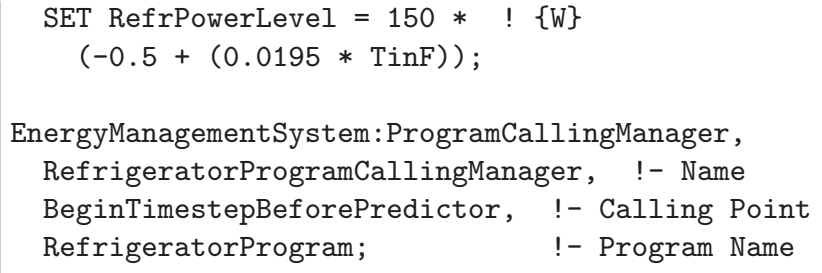

In CSE, every user input is an "actuator" with a defined variability: some inputs can vary hourly, some vary sub-hourly, and others can be defined only once per run (i.e., they are constant). The value of a an input can be set using an expression. Expressions include "probe" values (prefixed with an @), similar to the way EMS programs use sensor values. In CSE, every member of a record (including both inputs and outputs) can be a probe. The expression for any given input may include probes of equal or lesser variability (e.g., the expression for lighting power can include the zone's floor area, but not vice-versa). Expressions can also be used to describe time-of-year variability. In fact, there is no "schedule" object in CSE-if something varies every hour, you simply write an expression using the hour value (\$hour) or providing an array of 24 values to the built-in hourval () function. The same refrigerator example using expressions in CSE is shown below.

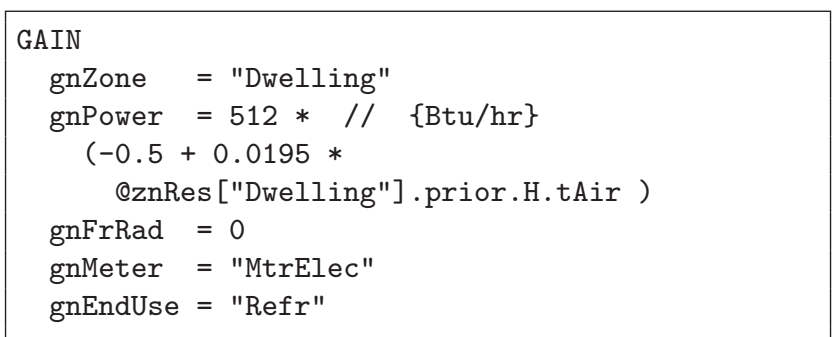

CSE expressions are an elegant way to provide user flexibility and is a core component of the program's software architecture. EMS was added later in EnergyPlus's development after the software architecture and input file syntax was already established. Both capabilities provide users with enhanced control over simulation behavior.

\section{Comparisons of results}

The two simulation engines were compared in the context of modeling real homes and prototype homes. All models were created and simulated by the same person to ensure consistent inputs. The models use practices representative of code compliance simulations in the State of California (e.g., 3-minute timesteps, and use of pressure-based airflow). These practices are not necessarily representative of all residential modeling applications. 


\section{Central Valley Research Homes}

The Central Valley Research Homes (CVRH) project monitored four Stockton, California homes of differing vintages (1948, 1953, 1996, and 2006) with wellcontrolled, repeatable indoor conditions. Detailed descriptions of the homes can be found in Proctor and Wilcox (2016). Roughly 130 data channels in each home provide minute-by-minute data of space temperatures, surface temperatures, component power, and local weather. The homes are unoccupied; occupantdriven latent and sensible heat gains are simulated using humidifiers and electric heaters. Data recording began in July, 2012 and is ongoing as of 2019. The comparisons presented here are based on data from July, 2012 to November, 2014.

Each home is equipped with an additional HVAC system with electric resistance heating, direct expansion cooling, and distribution within the conditioned space. Use of this "Reference System" allowed accurate measurement of thermal loads without system uncertainties. Thermostatic control of the home alternates every two days between the "Reference System" and a typical "House System" installation.

Part way through data collection, the homes underwent deep retrofits including changes to the air distribution system, higher efficiency HVAC equipment, wall and attic insulation, air sealing, and window replacements. Each of the homes were modeled pre- and post-retrofit in both CSE and EnergyPlus with and without the Reference System running.

Simulation results were compared for two of the homes (Grange and Mayfair) on the basis of total daily heating or cooling energy. Comparisons against the measured data from the CVRH project are based on total daily energy use for cooling and heating. Aggregate results are presented as Mean Absolute Error (MAE) and Mean Bias Error (MBE). The results in Figures 1 4 show close agreement between CSE and EnergyPlus, with notable examples where each simulation engine provided better agreement with the measured data; however, neither engine demonstrated consistently better agreement than the other. Table 1 shows a summary across both homes.

Table 1: Mean Absolute Error (MAE) and Mean Bias Error (MBE) in kWh/day (across both homes).

\begin{tabular}{lllll}
\hline End Use & CSE MAE & CSE MBE & EnergyPlus MAE & EnergyPlus MBE
\end{tabular}

\begin{tabular}{lllll}
\hline Heating & 6.21 & 3.95 & 5.52 & -2.42 \\
Cooling & 0.44 & 0.20 & 0.49 & -0.13 \\
\hline
\end{tabular}

\section{Prototypes}

Further comparison of algorithmic differences was conducted in the context of four prototype residential

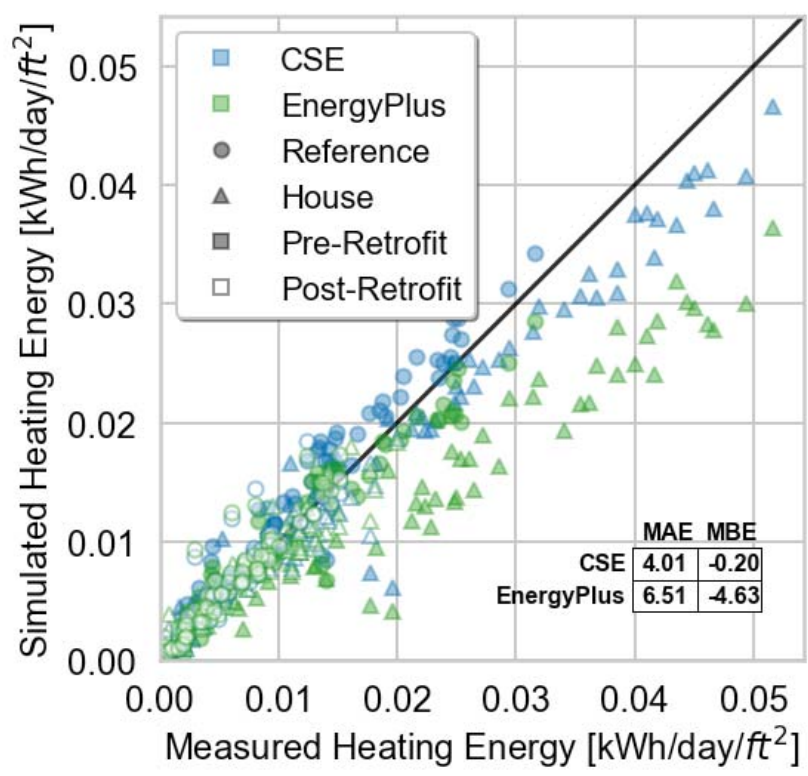

Figure 1: Grange Heating Comparison.

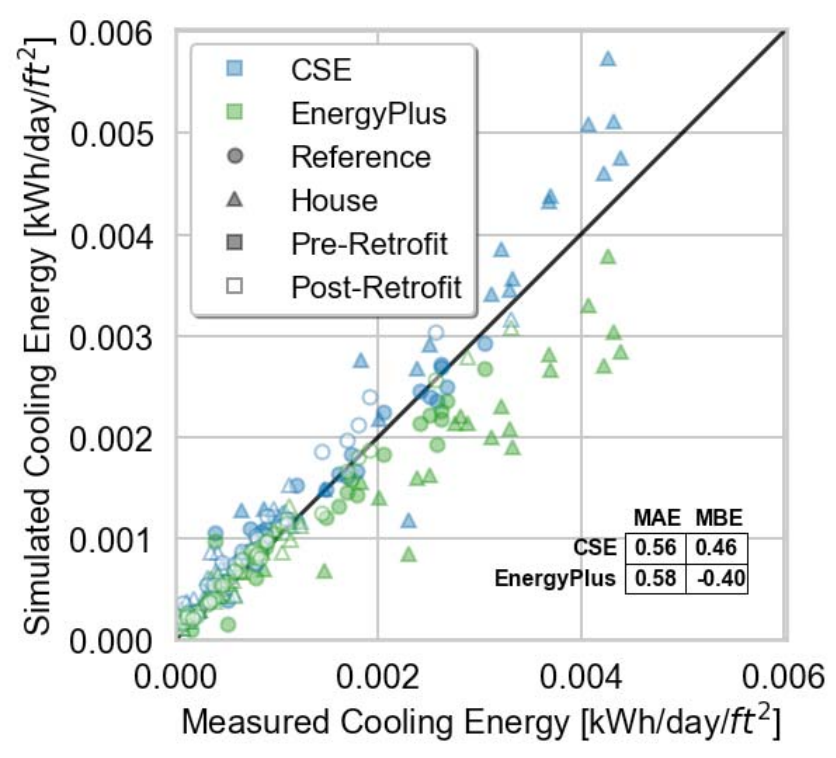

Figure 2: Grange Cooling Comparison.

building models: One Story, Two Story, Multifamily, and Townhome.

Prototype models offer a simplified and idealized environment to compare the simulation engines within a larger parameter space. Weather for California's Climate Zone 12 (Sacramento) was used for all prototype simulations.

\section{Temperature-dependent conductivity}

Temperature-dependent conductivity of materials is part of CSE's default conduction algorithm. EnergyPlus has the same capability, but requires the use of 


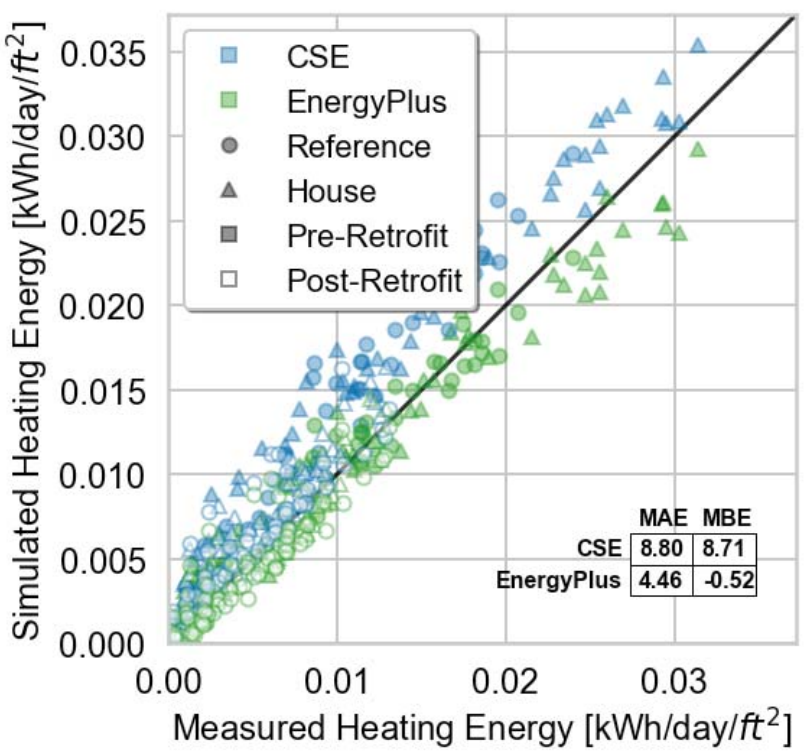

Figure 3: Mayfair Heating Comparison.

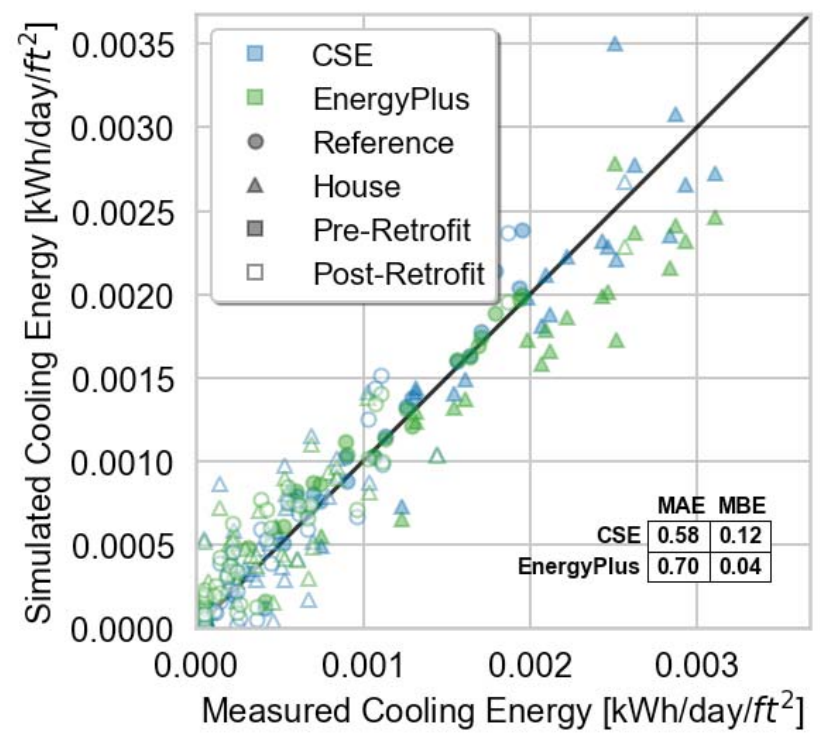

Figure 4: Mayfair Cooling Comparison.

its more advanced, finite difference model. Accounting for variable thermal conductivity of insulation materials is required for energy code compliance calculations in California.

The four prototype models were used to compare the effect of temperature-dependent conductivity of wall insulation for each prototype building in CSE and EnergyPlus. Results for annual heating and cooling energy are shown in Figure 5 and simulation runtime is shown in Figure 6.

While the variable thermal conductivity does not show a large impact on annual energy, the impact is typi-

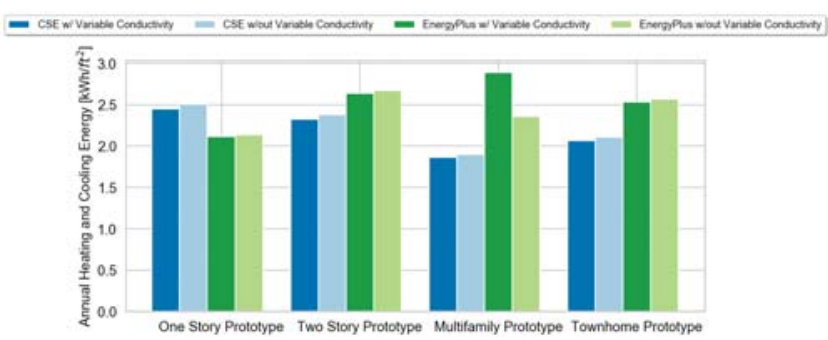

Figure 5: Prototype heating and cooling energy sensitivity to variable conductivity.

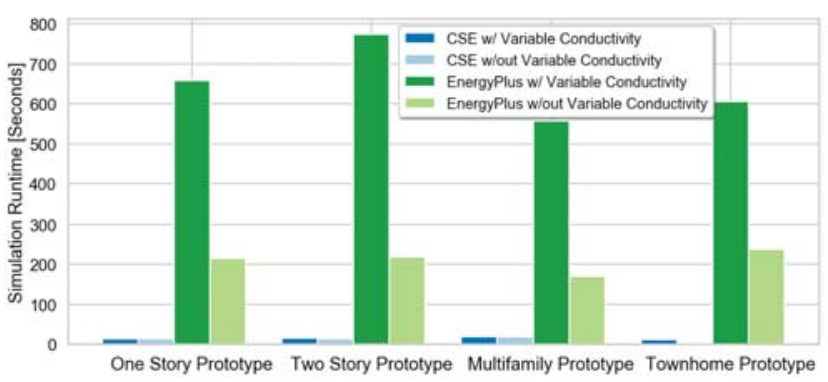
Figure 6: Prototype runtime sensitivity to variable conductivity.

cally greatest during the cooling season when electricity prices are much more volatile. Capturing the temporal impact of these loads on the greater electric grid is important.

\section{Timestep sensitivity}

CSE uses 3-minute timesteps for California energy code compliance analysis, while typical EnergyPlus simulations use 10- or 15-minute timesteps. For equivalent comparisons, all prototype models were compared using both 3- and 15-minute timesteps (using default conduction algorithms). Results for annual heating and cooling energy are shown in Figure 7 and simulation runtime is shown in Figure 8.

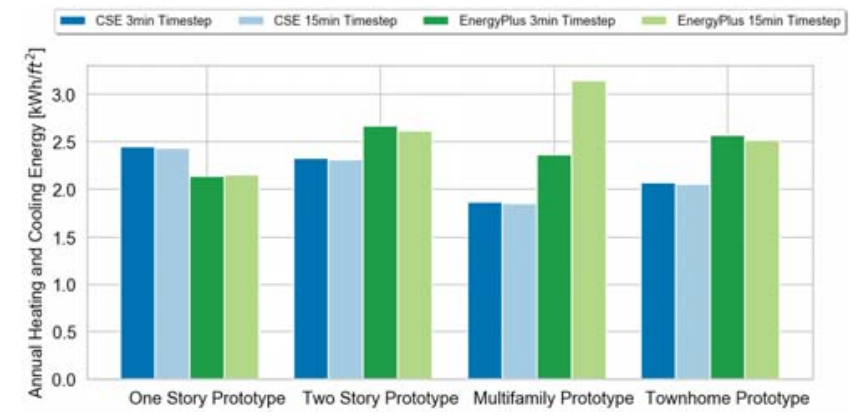

Figure 7: Prototype heating and cooling energy sensitivity to simulation timestep. 


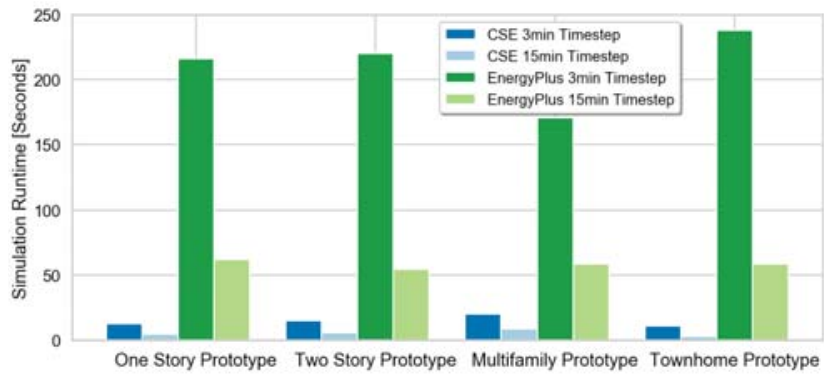

Figure 8: Prototype runtime sensitivity to simulation timestep.

\section{Conclusion}

CSE and EnergyPlus perform calculations at similar levels of algorithmic fidelity and offer comparable accuracy. EnergyPlus has significantly more simulation capabilities for non-traditional HVAC systems. CSE source code is based on object-oriented software design practices, which are less susceptible to inadvertent coding errors. CSE's input and expression syntax allows cleaner and more readable model definition.

The most notable difference between the two tools is the disparity in runtime with CSE simulations taking roughly 20 seconds each, and EnergyPlus taking roughly 300 seconds in the context of California code compliance. The disparity is exacerbated when using EnergyPlus's variable thermal conductivity algorithm (a requirement for compliance analysis California) resulting in runtimes up to 800 seconds - while CSE has a negligible change in runtime when using variable thermal conductivity.

\section{Disclaimers}

1. The lead author is a developer of both EnergyPlus and CSE. Other authors include developers of CSE, and users of both EnergyPlus and CSE.

2. Software changes rapidly. The content presented in this paper may be less relevant as developments are made to the respective simulation engines.

\section{References}

ASHRAE (2017). ANSI/ASHRAE Standard 140-2017: Standard Method of Test for the Evaluation of Building Energy Analysis Computer Programs.

Barnaby, C., B. Wilcox, and P. Niles (2013). Development and Validation of the California Simulation Engine. In Proceedings of Building Simulation 2013, Number August, Chambery, France, pp. 234-241. IBPSA.

Carroll, J. (1980). An 'MRT Method' of Computing Radiant Energy Exchange in Rooms. In Proceedings of the Second Systems Simulation and Economic Analysis Conference, San Diego, California.

Carroll, J. (1981). A Comparison of Radiant Interchange Algorithms. In Proc. ASME Solar Energy Division 3rd Annual Conf. on Systems Simulation, Economic Analysis/Solar Heating and Cooling Operational Results, Reno, Nevada.

California Energy Commision (2015). Residential Alternative Calculation Method Reference Manual for the 2016 Building Energy Efficiency Standards.

Horowitz, S., C. Dymond, E. Croteau, H. Khan, and R. Burns (2018). On the Need for a Unified and Collaboratively Developed Residential Building Simulation Platform. In 2018 ACEEE Summer Study on Energy Efficiency in Buildings, Pacific Grove, California. ACEEE.

Jones, N. and D. Greenberg (2011). Fast Computation of Incident Solar Radiation from Preliminary to Final Building Design. In Proceedings of Building Simulation 2011, Sydney, Australia, pp. 14-16. IBPSA.

Proctor, J. and B. Wilcox (2016). Deep Energy Retrofits - Lessons Learned from Central Valley Research Homes. In ACEEE Summer Study on Energy Efficiency in Buildings. ACEEE.

Roth, A., J. Bull, S. Criswell, P. Ellis, J. Glazer, D. Goldwasser, N. Kruis, A. Parker, S. Philip, and D. Reddy (2018). Scripting Frameworks for Enhancing EnergyPlus Modeling Productivity. In Proceedings of IBPSA-USA SimBuild 2018, Chicago, Illinois. IBPSA-USA.

The CSE Authors (2018a). CSE (California Simulation Engine). Version 0.856. https://github.com/cse$\operatorname{sim} /$ cse.

The CSE Authors (2018b). CSE User's Manual.

United States Department of Energy (DOE) (2018a). EnergyPlus: Engineering Reference. Berkeley, California.

United States Department of Energy (DOE) (2018b). EnergyPlus: Input Output Reference. Berkeley, California.

United States Department of Energy (DOE) (2018c). EnergyPlus. Version 9.0.1. https://github.com/NREL/EnergyPlus. 